\title{
Instalación de catéter venoso central por ultrasonido. Experiencia de la Unidad de Terapia Intensiva del Hospital Ángeles Pedregal
}

\author{
Alejandro Enriquez Vidal, ${ }^{1}$ Christopher Hernández Cortés, ${ }^{1}$ \\ Silvia del Carmen Carrillo Ramírez, ${ }^{2}$ Juan Gerardo Esponda Prado ${ }^{2}$
}

\section{Resumen}

La instalación de un catéter venoso central es una situación frecuente en las unidades de cuidados intensivos. En 1978 fue descrita la primera cateterización guiada por ultrasonido, demostrando así su efectividad y la disminución de las complicaciones mecánicas. Objetivo: Identificar la eficacia de la colocación de catéter venoso central con el uso de ultrasonido y las complicaciones atribuibles al procedimiento. Metodología: Estudio descriptivo, observacional y longitudinal en pacientes en quienes se intentó colocar un catéter venoso central yugular interno con ayuda del ultrasonido en la Unidad de Terapia Intensiva de Adultos del Hospital Ángeles Pedregal del 22 de septiembre de 2015 al 31 de julio de 2016. Se buscó la tasa de éxito y la identificación de las probables complicaciones inmediatas atribuibles al procedimiento. Resultados: El grupo de estudio se conformó de 35 pacientes. Fue exitosa la colocación de 33 de los 35 catéteres con una tasa de éxito de $94.28 \%$. Se determinó como causa de colocación no exitosa: colapso importante de la vena yugular interna y punción arterial. Conclusiones: El uso del ultrasonido para la colocación de un acceso venoso central es un procedimiento eficaz y con bajo índice de complicaciones.

Palabras clave: Catéter venoso central, ultrasonido.

\footnotetext{
${ }^{1}$ Médico Residente, Facultad Mexicana de Medicina, Universidad La Salle.

2 Médico adscrito a la Unidad de Terapia Intensiva de Adultos.
}

Hospital Ángeles Pedregal.

Correspondencia:

Dr. Alejandro Enriquez Vidal

Correo electrónico: alejandro_en_vi@hotmail.com

Aceptado: 26-10-2016.

Este artículo puede ser consultado en versión completa en http:// www.medigraphic.com/actamedica

\section{Summary}

The installation of a central venous catheter is a common situation in intensive care units. Since 1978 it was described the first ultrasound-guided catheterisation, thus demonstrating its effectiveness and decreased of mechanical complications. Objective: Identify the effectiveness of the placement of central venous catheter using ultrasound, and the complications attributable to the procedure. Methodology: Descriptive observational and longitudinal study of patients in which the placement of a central internal jugular venous catheter was attempted with the help of ultrasound in the Adult ICU of the Hospital Angeles Pedregal between September 22, 2015 and July 31,2016 . We sought to find the success rate and the identification of the likely immediate complications attributable to the procedure. Results: The study group was formed of 35 patients. The placement of 33 of the 35 were considered successful; success rate of $94.28 \%$. The major collapse of the internal jugular vein and an arterial puncture were determined as the reason for not successful placement. Conclusions: The use of ultrasound for the placement of central venous access is an effective and low complication rate procedure.

Key words: Central venous catheter, ultrasound.

\section{INTRODUCCIÓN}

La cateterización venosa se define como la inserción de un catéter biocompatible en el espacio intravascular, central o periférico, con el fin de administrar líquidos, fármacos, nutrición parenteral y determinar constantes fisiológicas. Su instalación en las unidades de cuidados intensivos es usual. ${ }^{1,2}$

Este procedimiento debe realizarse por personal capacitado. La técnica convencional de Seldinger descrita en 1953 y modificada es la más difundida y la de mayor aceptación. ${ }^{2,3}$ 
Desde 1978, año en que fue descrita la primera cateterización guiada por ultrasonido, se demostró tanto su efectividad como la disminución en las complicaciones mecánicas, logrando así reducir cada vez más las contraindicaciones absolutas para este procedimiento. 1,3,4

Dentro de la literatura se menciona como evidencia de grado $1 \mathrm{~A}$ el acceso yugular interno por ultrasonido y como grado $2 \mathrm{C}$ el acceso subclavio guiado. ${ }^{5-7}$

Las indicaciones y contraindicaciones para la instalación de un catéter venoso central se muestran en el cuadro 1.

La ubicación clásica descrita para la vena yugular interna es anterolateral en relación con la arteria carótida común en 9 a $92 \%$ de los casos, lateral en 0 a $84 \%$, anterior en 0 a $16 \%$, posterior en 0 a $9 \%$ y medial en 0 a $5.5 \% .{ }^{2,8}$ El diámetro promedio de la vena yugular interna es de $11.5 \mathrm{~mm}$, pero el diámetro mínimo se describe de $5 \mathrm{~mm}$ en 13 a 18\% de los sujetos. Existen además diferencias entre la vena yugular derecha e izquierda, siendo la primera más grande en $65 \%$ de los pacientes. ${ }^{2}$

El ultrasonido para la cateterización venosa central cuenta con múltiples estudios comparativos contra la punción a ciegas. Los aspectos descritos a favor del ultrasonido son: menor incidencia de punciones arteriales accidentales, menor cantidad de intentos fallidos, menor tiempo para efectuar el procedimiento, disminución en hematomas y reducción de riesgo de infecciones. ${ }^{1-3,9}$
Si bien algunas series describen el abordaje con ultrasonido por vena subclavia, aún no se realiza de rutina. Las series muestran entre 1.3 y 1.6 intentos para lograr acceder al sistema venoso con guía de ultrasonido., ${ }^{5,10}$ En un amplio estudio de 1,322 catéteres yugulares derechos instalados por ultrasonido se menciona una tasa de éxito de 96.9\%, con incidencia de $0.1 \%$ de neumotórax, sin punciones arteriales. ${ }^{5,6,10,11}$

Existen dos formas de abordaje por ultrasonido:

1. Abordaje estático: se visualiza la anatomía en forma previa y luego se procede a la punción.

2. Abordaje dinámico: se realiza en tiempo real, observando durante todo el procedimiento la inserción, siendo éste el más seguro y ampliamente utilizado por especialistas.

En un estudio se intentó observar las diferencias entre el corte longitudinal versus el corte transversal en la instalación del CVC, sin demostrar una diferencia significativa. ${ }^{4,10}$

Los beneficios e inconvenientes en la cateterización venosa guiada por ultrasonido fueron descritas por Ayoub C et al. (Cuadro 2). ${ }^{8}$

El uso del ultrasonido para el acceso venoso central ha llegado a ser tan seguro y fácil de realizar que varias series

\section{Cuadro 1. Indicaciones y contraindicaciones para la instalación de CVC}

$\begin{array}{ll}\text { Indicaciones } & \text { Contraindicaciones } \\ \text { Medición de presión venosa central } & \text { Contraindicaciones absolutas: } \\ & \text { - Rechazo del paciente } \\ \text { Requerimientos de múltiples infusiones en forma } & \text { - Contraindicaciones relativas: } \\ \text { simultánea } & \text { - Bulas pulmonares cuando se intenta el abordaje de la subclavia } \\ \text { Administración de drogas vasoactivas } & \text { - Hernia inguinal en accesos femorales } \\ \text { Quimioterapia } & \text { - Alteraciones carotídeas en accesos yugulares } \\ \text { Antibióticos tales como anfotericina-B e } & \text { - Paciente inquieto y no cooperador } \\ \text { infusiones hiperosmolares o por largos periodos } & \text { - Cuadros diarreicos cuando se considere el cateterismo de } \\ \text { Malos accesos venosos periféricos } & \text { venas femorales } \\ \text { Nutrición parenteral } & \text { - Traqueostomía con abundantes secreciones en abordajes } \\ \text { Procedimientos dialíticos } & \text { yugulares } \\ \text { Pacientes con riesgo potencial de instalar falla } & \text { - Punción de la subclavia y yugular izquierdas en pacientes } \\ \text { cardiovascular } & \text { - Trombosis de la vena }\end{array}$


mencionan que la duración del entrenamiento es de una hora a un día en residentes de urgencias, medicina interna y pediatría. Actualmente algunos sistemas educativos en Estados Unidos enseñan desde el pregrado su uso en modelos cadavéricos frescos para mejorar la destreza de los estudiantes. ${ }^{5,12}$

Nuestro estudio buscó demostrar la eficacia del ultrasonido para la instalación de accesos venosos centrales.

\section{METODOLOGÍA}

El estudio se desarrolló como descriptivo, observacional y longitudinal. Con el objetivo de evitar el sesgo por operador, se efectuaron dos intervenciones: la capacitación del personal y la estandarización de la técnica. La capacitación constó de un taller teórico-práctico de dos días en el que se explicó la forma de llevar a cabo el procedimiento, así como sus principales obstáculos y complicaciones. Aprobar este curso fue indispensable para ser considerado capacitado para la colocación de catéter venoso central por medio de ultrasonido. Para este estudio sólo se tomaron en cuenta los intentos para la colocación de catéter venoso central por ultrasonido efectuado por personal capacitado.

La técnica estandarizada consistió en visión directa a una o a dos manos del vaso por ultrasonido, con identificación de estructuras por compresión de vena yugular interna y en corte transversal, punción y paso de guía.

Se tomó como criterio de inclusión a todos los pacientes en quienes se intentó colocar un catéter venoso central yugular interno por medio de ultrasonido en la Unidad de Terapia Intensiva de Adultos del Hospital Ángeles Pedregal entre el 22 de septiembre de 2015 y el 31 de julio de 2016 por medio de personal capacitado. La indicación de colocación de catéter venoso central se dejó a criterio de los médicos tratantes. Fue indispensable contar con el consentimiento por escrito del paciente. Se excluyeron pacientes en quienes el primer intento fue sin uso de ul- trasonido. Finalmente, se descartaron aquéllos en quienes no hubo un seguimiento completo durante las primeras 24 horas después del intento de colocación del mismo.

Se consideró colocación exitosa la adecuada instalación del catéter venoso central utilizando el ultrasonido de forma dinámica, con acceso directo a la vena yugular interna, adecuado retorno en cada una de sus vías y sin lesión accidental a estructuras anatómicas vecinas.

Asimismo, una colocación no exitosa es aquélla en la que no se logró el acceso venoso o en la que hubo lesión de estructuras vecinas. En caso de no ser exitosa, se especificó la causa principal por la cual no se logró el acceso.

El seguimiento consistió en la evaluación e identificación de las probables complicaciones atribuibles al procedimiento. La identificación de las complicaciones se hizo de manera repetida durante las primeras cuatro horas posteriores al procedimiento, así como con una reevaluación después de 12 y 24 horas. Las complicaciones que se trató de identificar se muestran en el cuadro 3. Todos los datos fueron asentados en hojas de recolección para su análisis posterior.

Se utilizó la estadística descriptiva con medidas de tendencia central y de dispersión para describir las características generales del grupo de estudio, así como de la efectividad y las complicaciones del procedimiento. Para el análisis estadístico se utilizó el programa SPSS v19.

\section{RESULTADOS}

El grupo de estudio se conformó de 35 pacientes que cumplieron con los criterios para colocar un catéter venoso central por medio de ultrasonido. De ellos, $42.9 \%(n=15)$ eran del sexo masculino y $57.1 \%(n=20)$ del sexo femenino. Los pacientes presentaron edades de 31 a 95 años (rango de 64), con una mediana de 65 años (percentil 25 de 55 años y percentil 75 de 73 años). Asimismo, tenían una talla de 1.45-1.89 m (rango de $0.44 \mathrm{~m}$ ) con una mediana de 1.66 m (percentil 25 de 1.59 m y 75 de 1.74 m). Peso de 45 a

Cuadro 2. Beneficios e inconvenientes de la instalación de CVC guiada por ultrasonido.

Beneficios
Visualización de las estructuras vasculares
Ubicación óptima de la aguja
Protección ante la perforación de la pared posterior de
la VYI
Ubicación precisa del catéter
Disminución en el tiempo del procedimiento
Disminución en el número de intentos
Baja tasa de complicaciones

$$
\text { Inconvenientes }
$$

Necesidad de tecnología

Pérdida de habilidades técnicas usando puntos anatómicos

Falsa sensación de seguridad

Aumento en el costo de los equipos 
$130 \mathrm{~kg}$ (rango de 85) con una mediana de 76 kg (percentil 25 de $60 \mathrm{~kg}$ y 75 de $85 \mathrm{~kg}$ ). Con respecto al índice de masa corporal (IMC) presentaron un IMC de 19.7 a $37.7 \mathrm{~kg} / \mathrm{m}^{2}$ (rango de 18.1) con una mediana de 26.12 (percentil 25 de $22.15 \mathrm{~kg} / \mathrm{m}^{2}$ y 75 de $34.60 \mathrm{~kg} / \mathrm{m}^{2}$ ). Estratificando a los pacientes según su IMC nos encontramos que 37.1\% (13) tenía un peso normal, seguido por sobrepeso 31.4\% (11) y obesidad $31.4 \%$ (11). Ningún paciente de nuestro grupo de estudio mostró bajo peso.

El diagnóstico principal de los pacientes al momento de ingreso fueron los siguientes: cirugía de tracto digestivo (9; $25.7 \%)$, choque hipovolémico $(5 ; 14.3 \%)$, insuficiencia respiratoria $(9 ; 25.7 \%)$, choque séptico $(7 ; 20 \%)$, insuficiencia renal $(3 ; 8.6 \%)$ y abdomen agudo $(1 ; 2.9 \%)$.
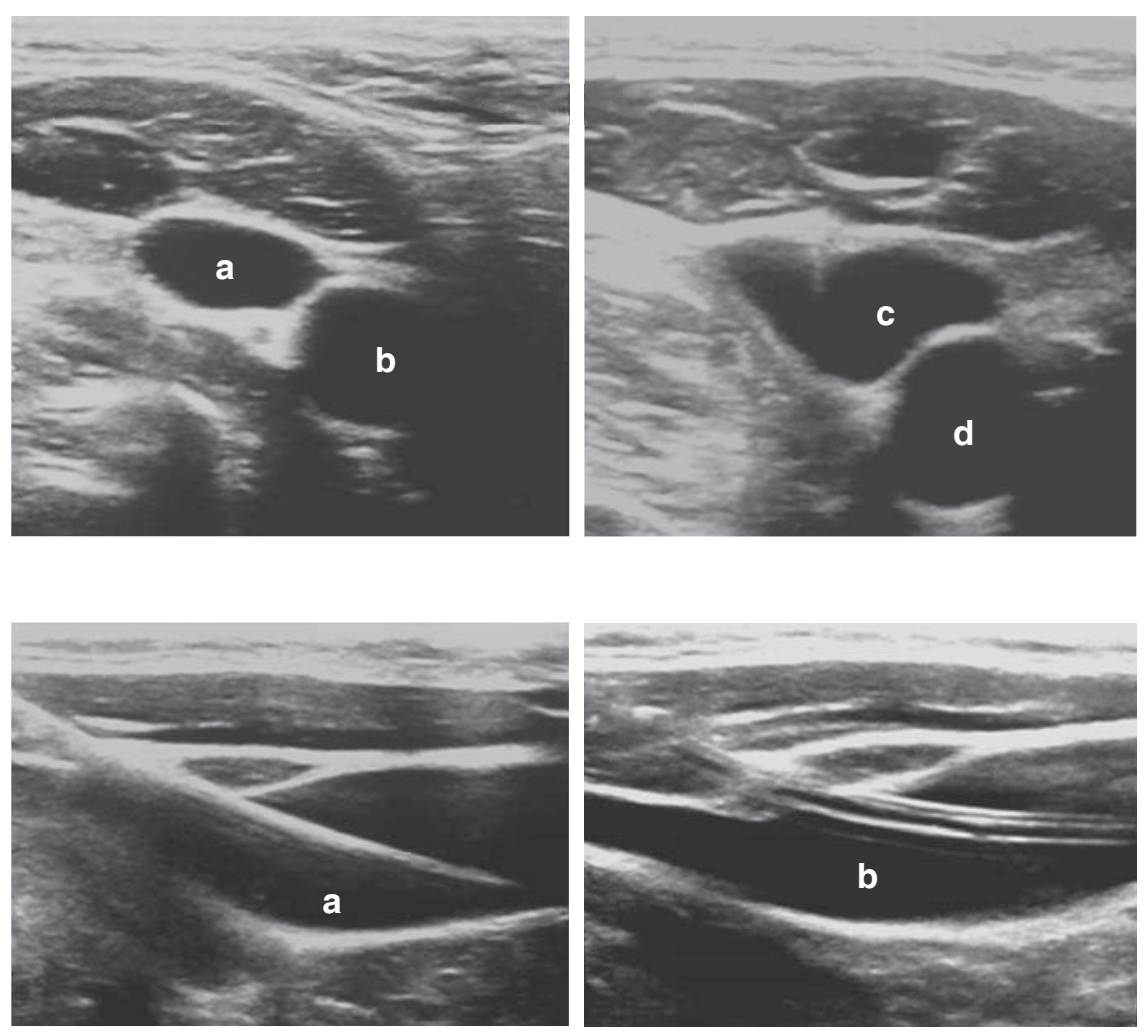

Figura 1.

Corte transversal. a. Vena yugular interna derecha (posición anterolateral). b. Arteria carótida común derecha. c. Vena yugular interna derecha en colapso por presión. d. Arteria carótida común derecha.

Figura 2.

Corte longitudinal. a. Paso de guía a través de vena yugular interna. b. Catéter venoso central en luz de vena yugular interna.

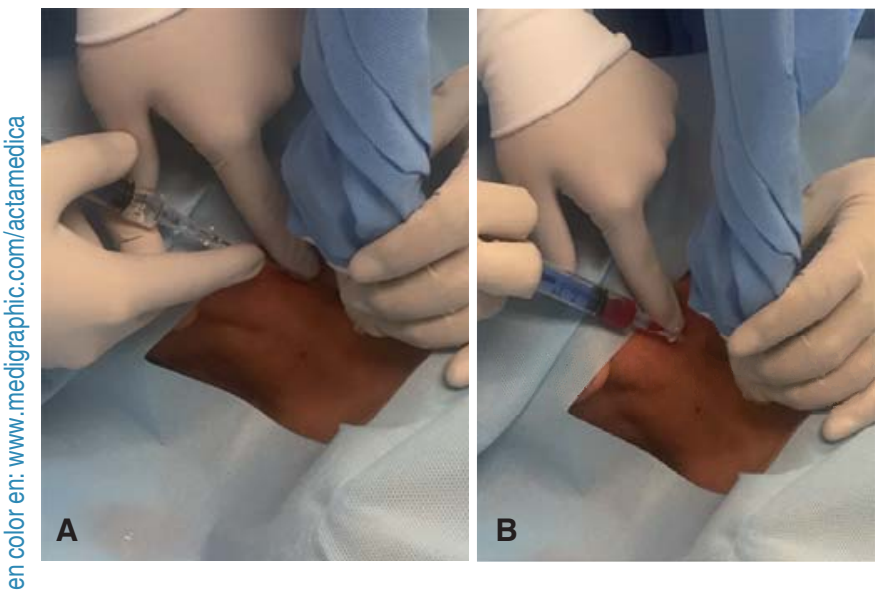

Figura 3. Punción guiada con ultrasonido con dos operadores. A. Punción en foco de la vena yugular interna derecha. B. Obtención de retorno al acceder a luz de vena yugular interna.

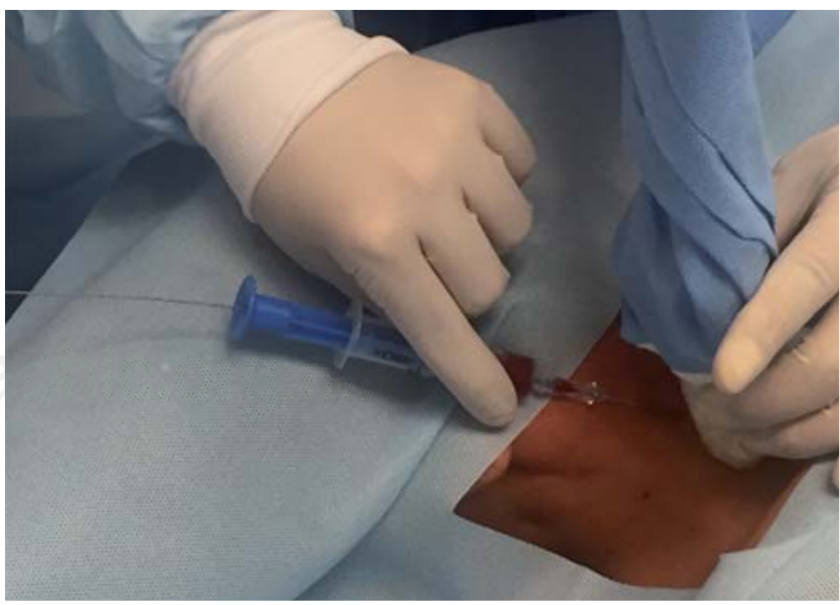

Figura 4. Paso de guía con ultrasonido. El paso de la guía del catéter debe visualizarse de forma directa en el ultrasonido. 


\begin{tabular}{|c|c|}
\hline \multicolumn{2}{|c|}{ Cuadro 3. Complicaciones evaluadas. } \\
\hline Falla en la canalización & Flebitis no infecciosa \\
\hline Neumotórax & Lesiones nerviosas \\
\hline Punción arterial & $\begin{array}{l}\text { Perforación de ca- } \\
\text { vidades cardiacas y } \\
\text { grandes vasos }\end{array}$ \\
\hline Punción del conducto torácico & Hemopericardio \\
\hline Perforación pleural & Rotura del catéter \\
\hline Trayectos aberrantes & Erosión cutánea \\
\hline Perforación mediastínica & \\
\hline Extravasación & \\
\hline Malfunción & \\
\hline Sangrado por el sitio de salida & \\
\hline
\end{tabular}

Se consideró exitosa la colocación de 33 de los 35 catéteres por ultrasonido con una tasa de éxito de $94.28 \%$.

En dos ocasiones no se logró colocar el catéter de forma exitosa. Se determinó como causa determinante de colocación no exitosa: el colapso importante de la vena yugular interna y la punción arterial (arteria carótida común izquierda). Finalmente, en ambos casos se aplicó la colocación del catéter sin la ayuda de ultrasonido, sin complicaciones.

La gran mayoría de los procedimientos se llevaron a cabo sin complicaciones inmediatas, después de 2, 4, 12 y 24 horas. La aparición de complicaciones de manera inmediata (dentro de las primeras 4 horas) fueron punción arterial $(n=1)$ y hemotórax $(n=1)$. No se detectaron nuevas complicaciones después de 12 y 24 horas del procedimiento.

\section{DISCUSIÓN DE RESULTADOS}

Los avances en la tecnología han logrado colocar el ultrasonido al alcance de las unidades de terapia intensiva con su implementación en la instalación de accesos vasculares, reduciendo así el riesgo de complicaciones. ${ }^{1,6,10}$

El uso del ultrasonido mostró una tasa de eficacia muy alta en nuestra serie (94.28\%), con bajo índice de complicaciones. Si bien este tipo de estudio se ha realizado en múltiples ocasiones, la experiencia en grupos mexicanos continúa siendo limitada.

Un aspecto que nos pareció destacable es el hecho de que gran parte de nuestro grupo de estudio se conformó de pacientes con sobrepeso y obesidad $(n=22)$ con una tasa de éxito en estos pacientes de 100\%, lo que confirma la seguridad y eficacia de este método en pacientes con esta característica, lo cual coincide con lo ya descrito en varios estudios. ${ }^{9}$

Aunque nuestro estudio no evaluó la identificación de complicaciones por medio del ultrasonido o su eficacia en comparación con la radiografía de tórax, consideramos que identificar complicaciones pudiese ser un uso adicional del ultrasonido, mientras que la radiografía de tórax se utiliza con el fin de identificar una normoposición de la punta del catéter.

\section{CONCLUSIONES}

El uso de ultrasonido para la colocación de un acceso venoso central es un procedimiento eficaz con un bajo índice de complicaciones.

La capacitación de dicho procedimiento es esencial para garantizar una óptima atención a los pacientes, si bien la curva de aprendizaje no termina, nuestra opinión es que el uso del ultrasonido para la instalación de un acceso venoso central yugular es un método sencillo, seguro y con alta tasa de efectividad.

\section{REFERENCIAS}

1. Miller AH, Roth BA, Mills TJ, Woody JR, Longmoor CE, Foster B. Ultrasound guidance versus the landmark technique for the placement of central venous catheters in the emergency department. Acad Emerg Med. 2002; 9 (8): 800-805.

2. Imigo F, Elgueta A, Castillo E, Celedón E, Fonfach C, Lavanderos J et al. Accesos venosos centrales. Cuad Cir. 2011; 25 (1): 52-58.

3. Durán-Briones G. Angioacceso central guiado por ultrasonografía de alta resolución para monitoreo invasivo transanestésico. Cir Cir. 2010; 78 (5): 418-422.

4. Vogel JA, Haukoos JS, Erickson CL, Liao MM, Theoret J, Sanz GE et al. Is long-axis view superior to short-axis view in ultrasound-guided central venous catheterization? Crit Care Med. 2015; 43 (4): 832-839.

5. Hourmozdi JJ, Markin A, Johnson B, Fleming PR, Miller JB. Routine chest radiography is not necessary after ultrasound-guided right internal jugular vein catheterization. Crit Care Med. 2016; 44 (9): e804-e808.

6. Zhong X, Hamill M, Collier B, Bradburn E, Ferrara J. Dynamic multiplanar real time ultrasound guided infraclavicular subclavian vein catheterization. Am Surg. 2015; 81 (6): 621-625.

7. Frankel HL, Kirkpatrick AW, Elbarbary M, Blaivas M, Desai H, Evans D et al. Guidelines for the appropriate use of bedside general and cardiac ultrasonography in the evaluation of critically ill patients-part I: general ultrasonography. Crit Care Med. 2015; 43 (11): 2479-2502.

8. Ayoub C, Lavallée C, Denault A. Ultrasound guidance for internal jugular vein cannulation: continuing professional development. Can J Anaesth. 2010; 57 (5): 500-514.

9. Raffán-Sanabria F, García-Acevedo MT, Celis E, Chaves A, RamírezPerdomo F, Díaz JC. Algoritmo de práctica clínica basado en la evidencia para el uso de ultrasonido en la colocación de catéteres venosos centrales. Rev Colomb Anestesiol. 2005; 33 (1): 51-58.

10. Schmidt GA, Maizel J, Slama M. Ultrasound-guided central venous access: what's new? Intensive Care Med. 2015; 41 (4): 705-707.

11. Biffi R, Bertoglio S, Pittiruti M; GAVeCeLT (Italian Study Group on Long-Term Central Venous Access). Insertion of central venous catheters (CVCs): any changes in the past 10 years? Ann Oncol. 2016; 27 (7): 1351.

12. Miller R, Ho H, Ng V, Tran M, Rappaport D, Rappaport WJ et al. Introducing a fresh cadaver model for ultrasound-guided central venous access training in undergraduate medical education. West J Emerg Med. 2016; 17 (3): 362-366. 